\title{
Novel two-step derivation method for the synchronous analysis of inherited metabolic disorders using urine
}

\author{
XIAO-QI SHENG ${ }^{*}$ and YI-CHAO WANG* \\ Hunan Province Technical Institute of Clinical Preventive and Treatment for Children's Inherited Metabolic Disorders, \\ Maternal and Child Health Hospital of Hunan Province, Changsha, Hunan 410008, P.R. China
}

Received June 30, 2015; Accepted January 3, 2017

DOI: $10.3892 /$ etm.2017.4167

\begin{abstract}
The aim of the present study was to conduct preliminary clinical screening and monitoring using a novel two-step derivatization process of urine in five categories of inherited metabolic disease (IMD). Urine samples (100 $\mu \mathrm{l}$, containing $2.5 \mathrm{mmol} / \mathrm{l}$ creatinine) were taken from patients with IMDs. The collected urine was then treated using a two-step derivatization method (with oximation and silylation at room temperature), where urea and protein were removed. In the first step of the derivatization, $\alpha$-ketoacids and $\alpha$-aldehyde acids were prepared by oximation using novel oximation reagents. The second-step of the derivatization was that residues were silylated for analysis. Urine samples were examined using gas chromatography/mass spectrometry (GC/MS) and a retention time-locking technique. The simultaneous analysis and identification of $>400$ metabolites in $>130$ types of IMD was possible from the GC/MS results, where the IMDs included phenylketonuria, ornithine transcarbamylase deficiency, neonatal intrahepatic cholestasis caused by citrin deficiency, $\beta$-ureidopropionase deficiency and mitochondrial metabolic disorders. This method was demonstrated to have good repeatability. Considering $\alpha$-ketoglutarate $(\alpha-K G)$ as an example, the relative standard deviations (RSDs) of the $\alpha-\mathrm{KG}$ retention time and peak area were 0.8 and $3.9 \%$, respectively, the blank spiked recovery rate was between 89.6 and $99.8 \%$, and the RSD was $\leq 7.5 \%$ $(n=5)$. The method facilitates the analysis of thermally non-stable and semi-volatile metabolites in urine, and greatly expands the range of materials that can be synchronously
\end{abstract}

Correspondence to: Professor Xiao-Qi Sheng, Hunan Province Technical Institute of Clinical Preventive and Treatment for Children's Inherited Metabolic Disorders, Maternal and Child Health Care Hospital of Hunan Province, 233 Xiangchun Road, Changsha, Hunan 410008, P.R. China

E-mail: lisaoydwxy@126.com

*Contributed equally

Key words: two-step derivatization, inherited metabolic disorders, simultaneous, urine, biomarker screened by GC/MS. Furthermore, it provides a comprehensive, effective and reliable biochemical analysis platform for the pathological research of IMDs.

\section{Introduction}

Inherited metabolic disorders (IMD) result from mutations of genes associated with metabolism, cofactors, channels, carriers or receptors in metabolic pathways, which are the basis of metabolic disorders of anabolic or catabolic pathways $(1,2)$. These mutations may be responsible for different types of biochemical abnormalities, including the accumulation of intermediate and/or bypass metabolites, or a deficiency of terminal metabolites, leading to metabolic crises, such as lactic acidosis, hyperammonemia syndrome and ketoacidosis $(3,4)$. Biochemical abnormalities can be classified as those involving organic acids, amino acids, fatty acids, carbohydrates, purines and pyrimidines. Genotype-phenotype correlations based on expression analysis of structural and functional mutations are gradually being recognized in different IMDs and subtypes of the disease, and >500 types of IMD have been identified so far (5). In all regions, it is reported that the overall incidence of neonatals with IMD is 1 in 1,000-2,500 newborns, while an estimated 16-20 million newborns are confirmed in China each year, indicating that effective and applicable measures are warranted due to the high-risk of infant IMD (6).

The majority of the abnormal metabolites associated with IMD are excreted in the urine; urine samples are easy to collect noninvasively without intrusive operations, so are therefore favored by researchers worldwide (7). Currently, the cross-association of substances with different diseases requires a preferable strategy to concurrently analyze characteristic metabolites in urine. However, expensive analytical instruments in combination with the long established 'modified urease pretreatment method' (8) and barriers to the fourth generation chemical analysis methodology have seriously hampered clinical and scientific development associated with IMD in China (9-11). Metabolomics is a new systematic approach for the study of changes in all components of intermediate molecular weight and terminal metabolites $(<1,000$ $M_{r}$ ) (12) for the early screening and monitoring of IMD in children. Metabolomics is characterized by high throughput, sensitivity and selectivity. The simultaneous analysis of metabolites in urine and the study of various types of IMD 
have become popular research topics. As an advantageous analytical tool, gas chromatography/mass spectrometry (GC/ MS) is able to analyze a variety of complex biological materials, including many metabolites that are volatile and thermally stable (13-15). Furthermore, GC/MS with retention locking time techniques (GC-MS-RTL) and associated software have provided a strong foundation for metabolomics research (16). However, related sample pretreatment technology has become a limitation that requires investigation. The GC/MS technique is limited for the analysis of thermally non-stable amino acids, $\alpha$-ketoacids and $\alpha$-aldehyde acids, volatile and semi-volatile organic acids, and polar metabolites.

Clinical technologies for simultaneous testing have been reported for different types of materials, diseases and small molecules in the urine $(17,18)$. However, simultaneous analysis procedures for the clinical analysis of urine have rarely been reported for the hundreds of IMDs in China. The substances in urine comprise five categories of small molecules with differing compositions, properties and structure. A two-step chemical derivatization method (19) for urine pretreatment has achieved the ability to synchronously screen organic acids, fatty acids, carbohydrates, purines and pyrimidines. The clinical significance of this two-step chemical derivatization method merits exploration in children with IMD.

The present study was performed to identify clinical screening and monitoring values for five categories of IMDs using the self-developed two-step derivatization method in urine. The study aimed to explore potential markers for distinguishing phenylketonuria, ornithine transcarbamylase (OTC) deficiency, neonatal intrahepatic cholestasis caused by citrin deficiency (NICCD), $\beta$-ureidopropionase deficiency, and mitochondrial metabolic disorders with $8993 \mathrm{~T}>\mathrm{G}$ mutants from control subjects. The method enabled the simultaneous examination of urinary organic acids (including $\alpha$-ketoacids and $\alpha$-aldehyde acids), amino acids, fatty acids, carbohydrates, purines and pyrimidines in $100-\mu 1$ urine samples.

\section{Materials and methods}

Ethics statement. The protocol of the current study has been approved by the Ethics Committee of the Hunan Province Technical Institute of Clinical Preventive and Treatment for Children's IMDs (Changsha, China). Written informed consent was obtained from each child's parents or guardians prior to the performance of the following experimental procedures.

Source of material. From October 2010 to May 2014, children with IMD-related symptoms treated in the Maternal and Child Health Hospital of Hunan Province were selected and enrolled in the study, including children who were stunted, or had mental retardation, jaundice delay, epilepsy or seizures, cerebral palsy and autism spectrum disorders. Inclusion criteria for those eligible children were as follows: i) Children with low birth weight, preterm children and severely malnourished IMD-risk children; ii) children with normal or abnormal liver and kidney function; and iii) children whose ages ranged between 0 and 12 years. The male to female ratio was $2.5: 1$. Morning urine and postprandial urine $(2.5 \mathrm{~h}$ after a meal $)$ samples $(3-10 \mathrm{ml})$ were separately collected and stored in a refrigerator at $-70^{\circ} \mathrm{C}$ (Haier Group, Qingdao, China).
The main focus of the research was the analysis of pathogenic genes, including those associated with phenylketonuria (c.782G $>$ A), OTC deficiency (c.912G $>$ T), NICCD (c.851-854del4; NG_012247.1:g.A92352A/G), thymine-uracil aciduria (c.C216C/T), $\beta$-urea acid deficiency diseases (c.C792C/A; c.G977G/A) and mitochondrial metabolic disorders $(\mathrm{m} .8993 \mathrm{~T}>\mathrm{G} ; \mathrm{m} .10191 \mathrm{~T}>\mathrm{C})$.

Sample pretreatment. Urine samples (100 $\mu \mathrm{l}$, containing $2.5 \mathrm{mmol} / \mathrm{l}$ creatinine) were added to $30.0 \mu \mathrm{l}(1.2 \mathrm{U} / \mu \mathrm{l})$ urease, and then incubated at $37^{\circ} \mathrm{C}$ for $\sim 30 \mathrm{~min}$. Following the addition of $50 \mu \mathrm{l}$ of the internal standards tropic acid solution and heptadecanoic acid solution $(0.5 \mathrm{mg} / \mathrm{ml}$, respectively; Sigma-Aldrich; Merck Millipore, Darmstadt, Germany) and mixing, $800 \mu 1$ ethanol was added, followed by centrifugation at $12,000 \times \mathrm{g}$ for $15 \mathrm{~min}$ at room temperature $\left(37^{\circ} \mathrm{C}\right)$. Subsequently, $40 \mu 1$ hydroxylamine hydrochloride solution $(0.04 \mathrm{~mol} / \mathrm{l})$ and $60 \mu \mathrm{l} \mathrm{Ba}(\mathrm{OH})_{2}$ solution $(0.05 \mathrm{~mol} / \mathrm{l})$ were supplied, and a solution of oxime compound was thus generated. The liquid was heated for $60 \mathrm{~min}$ at $60^{\circ} \mathrm{C}$, and the supernatant was extracted into a small sample preparation bottle placed under a nitrogen blowing instrument and carefully dried at $60^{\circ} \mathrm{C}$. The residue was then combined with $100 \mu 1$ double-derivatizing reagent N,O-bis(trimethylsilyl) trifluoroacetamide-trimethylchlorosilane (BSTFA-TMCS, 99:1; Sigma-Aldrich; Merck Millipore), $20 \mu \mathrm{l}$ pyridine co-solvent and other self-made silylated complex liquids [including BSTFA (bis-trimethylsilyl-trifluoroacetamide), MSTFA (N-methyl-trimethylsilyltrifluoroacetamide), MTBSTFA (N-(ter-Butyldimethylsilyl)-N-methyltrifluoroacetamide) and TMCS (trimethyl chlorosilane)], and then placed at $80^{\circ} \mathrm{C}$ under a drying heater to initiate the silanization derivatization process, as previously described (19). Subsequently, the derivatized products were transferred into a bottle, diluted to a volume of $500 \mu \mathrm{l}$, and $1.0 \mu \mathrm{l}$ derivatized products were taken for analysis.

GC/MS conditions. Instrumentation used in this procedure comprised a GC/MS instrument (7890-5975C Agilent Technologies, Inc., Santa Clara, CA, USA), with a J\&W HP-5 capillary column (column length, $60 \mathrm{~mm}$; film thickness, $25 \mathrm{~mm}$; inner diameter, $0.25 \mu \mathrm{m}$ ). The carrier gas was helium with a high purity $(99.995 \%)$ at a flow rate of $1.5 \mathrm{ml} / \mathrm{min}$; the inlet temperature was $250^{\circ} \mathrm{C}$, and the detailed temperature program was as follows: An initial column oven temperature of $60^{\circ} \mathrm{C}$ for $4 \mathrm{~min}$, followed by a $6-15^{\circ} \mathrm{C} / \mathrm{min}$ rate of temperature increase up to $320^{\circ} \mathrm{C}$ for $10 \mathrm{~min}$. The interface temperature was $300^{\circ} \mathrm{C}$, and the electronic ionization (EI) source energy was $70 \mathrm{eV}$ with an ion-source temperature of $230^{\circ} \mathrm{C}$.

Basic parameters of data processing. Using the SCAN mode, the retention times of the chromatographic peaks for tropic acid and heptadecanoic acid were determined.For tropic acid-TMS2, the retention time was $26.0340 \mathrm{~min}$ and its characteristic ion mass-to-charge ratios were 103, 115 and 280. Furthermore, for heptadecanoic acid-TMS1, the retention time was $36.1070 \mathrm{~min}$ and characteristic ion mass-to-charge ratios were 132, 145 and 327. Using the selecting ion scanning (SIM) mode for qualitative and quantitative analysis, drift in the retention time was automatically corrected by the software, using retention time 
locking technology. Therefore, the retention time of tropic acid TMS2 was locked at $29.9280 \pm 0.0100 \mathrm{~min}$ and that of heptadecanoic acid-TMS1 was locked at $36.0000 \pm 0.0100 \mathrm{~min}$; the ratio of five different peak areas for $80,90,100,110$ and $120 \mu \mathrm{l}$ of $12.9 \mathrm{mmol} / \mathrm{l}$ tropate to the peak area for $100 \mu \mathrm{l}$ of $2.5 \mathrm{mmol} / \mathrm{l}$ creatinine was used to create a calibration curve (metabolomic data normalization), as an internal standard method of quality control, as described in our two previously published papers $(8,20)$. The ratio of the peak areas of the metabolites to the peak area of $100 \mu \mathrm{l}$ of $2.5 \mathrm{mmol} / \mathrm{l}$ creatinine were used as parameters in a semi-quantitative analysis, in accordance with the establishment of a principal component analysis (PCA) model.

Pattern recognition results and the Physician's Guide to the Laboratory Diagnosis of Metabolic Diseases (21) were used to identify the metabolites.

Statistical analysis. Independent-sample t-tests were used for the comparison of the metabolite levels to identify significant differences between the preliminary study group and gender/age-matched controls. Statistical analysis of some characteristic metabolites has been described in detail in our previous papers $(8,20)$. A PCA model was constructed using differentially expressed compounds, with $\mathrm{P}<0.05$ considered to indicate a statistically significant difference. Deconvolution Reporting Software (version A.02.00), an Automated Mass Spectral Deconvolution and Identification System (5975C-7890A) and Mass Profiler Professional software (version 2.0; all Agilent Technologies, Inc.) were used.

\section{Results}

Simultaneous analysis using two-step derivatization in phenylalanine hydroxylase deficiency. The urine from patients with phenylketonuria (c.782G $>$ A) presented a wide range of peaks. Test results for patients with phenylketonuria indicated the presence of not only organic acids, but also amines, amino acids and fatty acids (Fig. 1). The urine metabolic spectrum indicated the presence of $\mathrm{N}$-acetylphenylalanine, tyrosine, phenylacetate, mandelate, $o$-hydroxyphenylacetate, phenyllactate, phenylpyruvate, phenylacetylglutamine and homovanillate. Thus, the present method was able to detect various metabolites in the urine simultaneously.

Simultaneous analysis using two-step derivatization in urea cycle disorders. The metabolites present in the urine from patients with OTC deficiency (c.912G $>$ T) were simultaneously presented under the current experimental conditions. The results identified the presence of uracil, orotate and uridine, among other metabolites, in the urine (Fig. 2).

Simultaneous analysis using two-step derivatization in citrin deficiency. In the urine samples from patients with citrin deficiency (c.851-854del4; NG_012247.1: g.A92352A/G) galactose, galactonate and galactitol were detected, which might be due to abnormal metabolic pathways for galactose, in addition to glucose, gluconate and glucuronate (glucose disorders), and 4-hydroxyphenylacetate, 4-hydroxyphenyllactate, 4-hydroxyphenylpyruvate, $\mathrm{N}$-acetyltyrosine, phenylalanine and phenylacetate (tyrosine and phenylalanine disorders). The

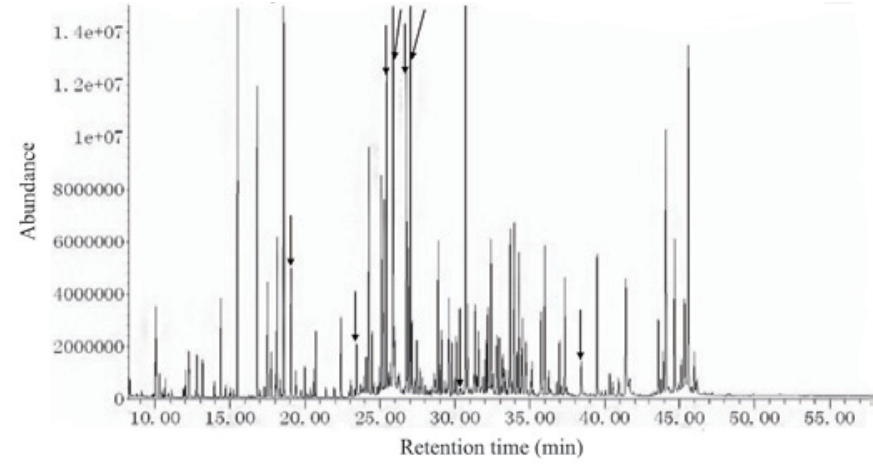

Figure 1. Urine metabolic spectrum from a patient with classic phenylketonuria (c.782G $>$ A). From left to right, peaks for phenylacetic acid, mandelic acid, ortho-hydroxyphenylacetic acid, phenyllactic acid, phenylalanine, phenylpyruvic acid, $\mathrm{N}$-acetylphenylalanine and phenylacetylglutamin (and its bypass metabolite products, including tyrosine, tryptophan, vitamins and secondary metabolities) are indicated by arrows. All the indicated peaks, with the exception of $\mathrm{N}$-acetylphenylalanine, were markedly increased.

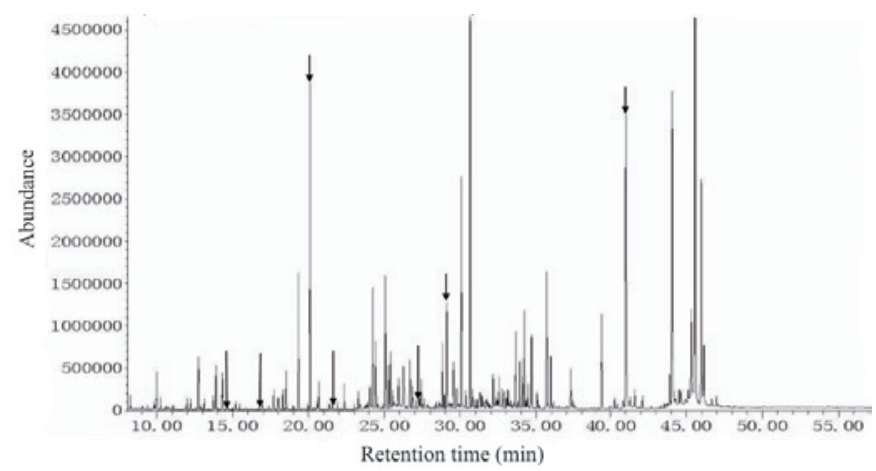

Figure 2. Urine metabolic spectrum from a patient with ornithine transcarbamylase deficiency (c.912.G>T). From left to right, peaks for $\beta$-alanine, $\beta$-aminoisobutyratic acid, uracil, thymine, $\beta$-ureidopropionic acid, orotic acid and uridine are indicated by arrows. Urine levels of uracil, orotic acid, and uridine were markedly increased.

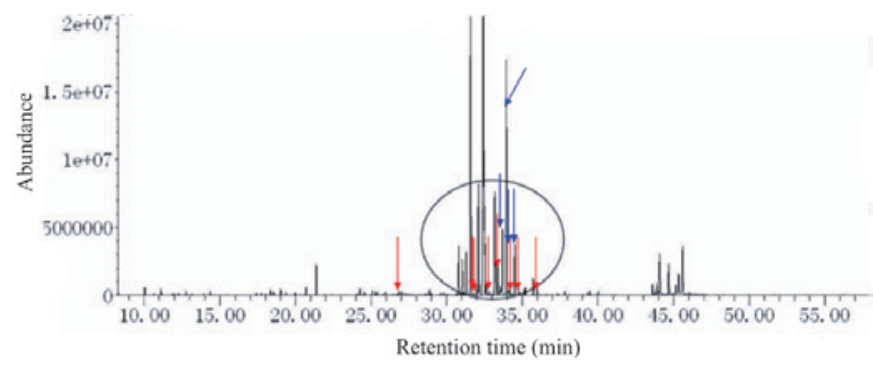

Figure 3. Urine metabolic spectrum from a patient with neonatal intrahepatic cholestasis caused by citrin deficiency (c.851-854de14). From left to right, peaks for 4-hydroxyphenylacetate, galactonate, 4-hydroxyphenyllactate, 4-hydroxyphenylpyruvate, galactitol, galactose-oxime-1, glucose-oxime-1, and glucose-oxime-2 are indicated by red arrows. Gluconate, glucuronate, galactose-oxime- 2 and $\mathrm{N}$-acetyltyrosine are indicated by blue arrows. The main biomarkers of the disorders are circled, excluding phenylalanine, tyrosine, methionine and ornithine. Urine levels of galactose-oxime-1 and galactose-oxime-2 were markedly increased.

urine metabolic spectrum (Fig. 3) showed peaks for galactose, galactonate, galactitol, glucose, gluconate, glucuronate, 4-hydroxyphenylacetate, 4-hydroxyphenyllactate, 4-hydroxyphenylpyruvate, tyrosine and $\mathrm{N}$-acetyltyrosine (Fig. 3). 


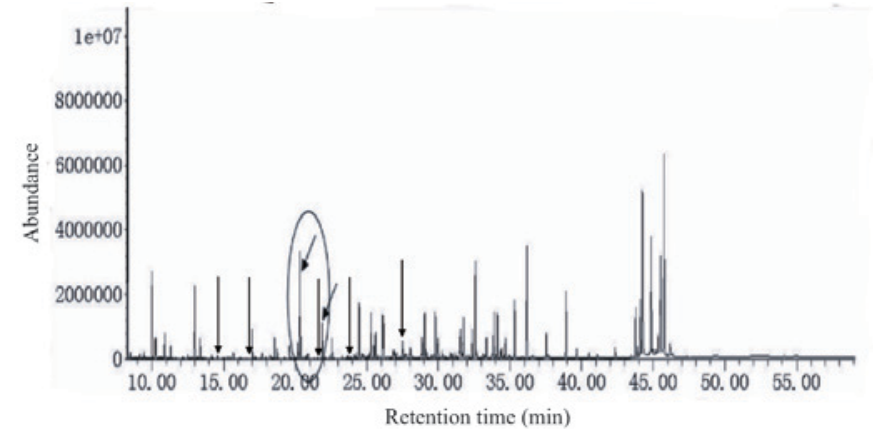

Figure 4. Urine metabolic spectrum from a patient with thymine-uracil aciduria (c.C216C>T) combined $\beta$-ureidopropionase deficiency (c.C792C/A and c.G977G/A). Peaks from left to right correspond to $\beta$-alanine, $\beta$-aminoisobutyric acid, uracil, dihydrouracil, thymine, dihydrothymine, and $\beta$-ureidopropionic acid (arrows). Urine levels of uracil, thymine and $\beta$-ureidopropionic acid were markedly increased.

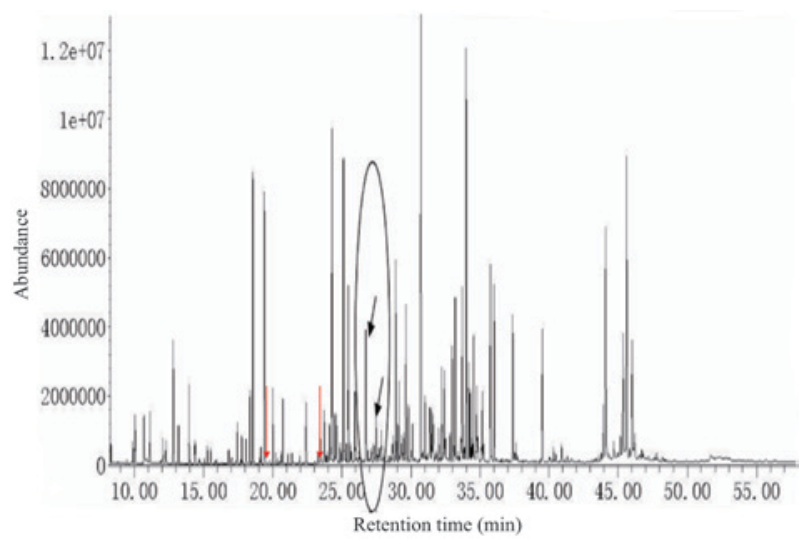

Figure 5. Urine metabolic spectrum from a case with MT-ATP6 (m.8993T>G) mutation. Peaks from left to right correspond to succinate-1, succinate-2 (red arrows), $\alpha$-ketoglutarate-oxime-1 and $\alpha$-ketoglutarate-oxime-2 (black arrows), and involve metabolites associated with the Krebs cycle.

Simultaneous analysis using two-step derivatization in purine and pyrimidine deficiencies. The urine from patients with thymine-uracil aciduria (c.C216C/T), $\beta$-ureidopropionase deficiency (c.C792C/A and c.G977G/ A) presented results under the current analytical conditions. The results indicated the presence of uracil, thymine, 5-hydroxymethyl-uracil, dihydrouracil, dihydrothymine, $\beta$-ureidopropionate, $\beta$-aminoisobutyrate and $\beta$-alanine, among others (Fig. 4). These results indicated that metabolites associated with the metabolic pathways of purines and pyrimidines can be detected appropriately using this two-step derivatization method.

Simultaneous analysis using two-step derivatization in mitochondrial metabolic disorders. The urine from patients with mitochondrial metabolic disorders (e.g., m..8993T $>$ G, m.10191T> C, m.14487 T>C, m.14693 A>G) was clearly presented under the current experimental conditions. Results which are closely correlated with mitochondrial metabolic disorders include lactate pyruvate, $\beta$-hydroxybutyrate, acetoacetate, ethylmalonate, methylmalonate, adipate, azelate and sebate were observed. In addition, $\alpha$-ketoglutarate $(\alpha-\mathrm{KG})$ and succinate were also detected (Fig. 5). Both $\alpha-K G$ and succi- nate were clearly increased to varying degrees in the urine of outpatients with mitochondrial metabolic disorders.

Methodological evaluation using two-step derivatization. Repeated injection of the same urine sample containing standards of $\alpha-\mathrm{KG}$ was conducted 9 times and the relative standard deviations (RSDs) of the retention time and peak area of $\alpha-\mathrm{KG}$ were found to be 3 and $1.3 \%$, respectively, indicating that the apparatus had a good level of precision. Following the preparation of 9 urine samples in parallel, the RSDs of the retention time and peak area of $\alpha-\mathrm{KG}$ were found to be 0.8 and $3.9 \%$, respectively, indicating that the reproducibility of the sample preparation method was also good. When a blank urine sample was taken, $\alpha-K G$ added and the recovery measured, the average recoveries were $93.1,99.8$ and $89.6 \%(\mathrm{n}=9)$, respectively, for $3.3788,6.7576$ and $10.1364 \mu \mathrm{mol} / \mathrm{l} \alpha-\mathrm{KG}$, and the RSD was $<7.5 \%(\mathrm{n}=9)$.

\section{Discussion}

Since this two-step derivatization method was devised, it has been applied to the analysis of $>3,320$ cases from the Hunan Province of China, and elsewhere. Our previous investigations supported the ability of two-step derivatization to simultaneously screen and monitor biochemical metabolic markers of five categories of IMDs $(8,22)$. At present, classic phenylketonuria, OTC deficiency diseases, citrin deficiency, $\beta$-ureidopropionase deficiency, and 8993T $>\mathrm{G}$ mitochondrial metabolic disorders have been detected, and 69 single diseases and 516 cases of children with IMDs, such as citrin deficiency disease (NG_012247.1 g.A92352A/G) and other genetic analyses have been confirmed with testing of gene mutations. We have detected the presence of one or more secondary metabolic diseases in some individuals, which has enabled a comprehensive understanding of their complications and effective treatment.

However, it difficult to investigate all aspects of metabolic diseases due to the wide variety of metabolites, namely amino acids, organic acids, fats, carbohydrates, purines and pyrimidines. Therefore, this study investigated classic phenylketonuria, OTC deficiency diseases, citrin deficiency, $\beta$-ureidopropionase deficiency and 8993T $>\mathrm{G}$ mitochondrial metabolic disorders, focusing on the analysis of relevant biomarkers using the two-step derivatization of urine samples.

There are two types of defects associated with phenylketonuria and hyperphenylalaninemia, which are correlated with the tetrahydrobiopterin (BH4) anabolic pathway or phenylalanine catabolic pathway $(23,24)$. A wide range of biomarkers for phenylketonuria (c.782G $>$ A) were presented in the present study, including phenylalanine, $\mathrm{N}$-acetylphenylalanine, tyrosine, phenylacetate, mandelate, $o$-hydroxyphenylacetate, phenyllactate, phenylpyruvate, phenylacetylglutamine and homovanillate. These biomarkers of phenylketonuria have been detected previously through mass spectrometry with electrospray ionization (15) and high-performance liquid chromatography with a GC-clamped primer combined with other analytical methods (25).

The present study focused on classical phenylketonuria, which has been described in detail in our previous study (20). Some biomarkers were identified as being helpful 
for secondary differential disorders in certain complications associated with classical phenylketonuria. These are increased 4-hydroxyphenylacetate, 4-hydroxyphenylpyruvate and 4-hydroxyphenyllactate (secondary tyrosinemia type III, or vitamin C deficiency), 5-hydroxyindoleacetate, 5-hydroxyindolepyruvate and 5-hydroxyindolepropionate (secondary tryptophan deficiency), lactate, pyruvate and alanine (vitamin B1 deficiency), glutarate, succinate and $\alpha-\mathrm{KG}$ (vitamin B2 deficiency), quinolinate, 4-hydroxyquinolinate and 4,8-dihydroxyquinolinate (vitamin B6 deficiency), and methylmalonate and 2-methylcitrate (vitamin B12 deficiency), respectively. Furthermore, various biomarkers were used to discern classical phenylketonuria (increased phenylacetate), hyperphenylalaninemia (increased phenylalanine) and BH4-defects (increased 5-hydroxyindoleacetate, 5-hydroxyindolepyruvate and 5-hydroxyindolepropionate). Thus, the analysis of urine samples using two-step derivatization pretreatment under optimal conditions enabled the screening of numerous potential biomarkers in phenylketonuria and hyperphenylalaninemia.

There are $>10$ types of defect in the urea cycle, which have been shown to be associated with various metabolic pathways (26-30). The activities of enzymes including $\mathrm{N}$-acetylglutamate synthetase (NAGs), carbamoyl phosphate synthetase I (CPS-I), argininosuccinate synthetase, argininosuccinate lyase, OTC and argininase are severely lacking in the urea cycle, leading to the accumulation of ammonia and other abnormal metabolites. When screening for the urea cycle and related disorders, there are two key challenges, which are the low solubility of the metabolites, and their sensitivity to oxidation and reduction. Most importantly, orotate is characterized by thermal non-stability, high sensitivity to oxidation and reduction and poor solubility in organic solvents, and so has not been precisely tested using conventional methods (31).

In the present study, at least six biomarkers were found to be helpful for severe OTC deficiency and its secondary $\beta$-ureidopropionase deficiency, including uracil, orotate, uridine, $\beta$-ureidopropionate, $\beta$-aminoisobutyrate and $\beta$-alanine. Reports of these OTC biomarkers in the literature are rare (32). In the present study, a PCA model was constructed using uracil, orotate and uridine, respectively.

In addition, CPS-I deficiency and NAG deficiency may cause hyperammonemia (33), Even though we did not find carbamyl phosphate accumulation in these two disorders, the present method was able to detect elevated glutamine, alanine and asparagine, combined with hyperammonemia, so as to enable a differential diagnosis.

Citrin deficiency is a defect of mitochondrial carrier protein glutamate-aspartate carrier that was discovered in 1999 (34). Citrin has several functions, which include participation in the biosynthesis of urea, protein and nucleic acids (35), and the provision of adequate NADH for mitochondrial membranes (36). Therefore, citrin dysfunction affects synthetic or catabolic pathways, leading to a series of metabolic dysfunctions and varying degrees of clinical manifestations (37). The most common mutants associated with citrin deficiency have been identified (c.1177+1G $>$ A and 851-854del) (38).

In general, three critical metabolic pathways were identified as potential biomarkers for citrin deficiency in the present study: Galactose, galactonate and galactitol; glucose, gluconate and glucuronate; and 4-hydroxyphenylacetate, 4-hydroxyphenyllactate and 4-hydroxyphenylpyruvate. Differences in their expression was detected between classical patients and normal controls, with a PCA model constructed using these biomarkers. Notably, 4-hydroxyphenyllactate and 4-hydroxyphenylpyruvate are readily oxidized and reduced metabolites.

Other biomarkers, such as methionine, lysine, threonine, citrulline, arginine and ornithine, for non-classical or classical citrin deficiency have been reported in previous studies $(39,40)$. However, lysine, citrulline and ornithine are characterized by a lack of thermal stability, and ease of oxidation and reduction and, to the best of our knowledge, have not been precisely tested using conventional derivatization. With respect to derivatization, in the present study the urine samples were pretreated at room temperature, and minute $\mathrm{pH}$-value variation was required to obtain optimal conditions. Furthermore, the present study newly identified certain metabolites of phenylacetate, $\mathrm{N}$-acetyltyrosine, adenine and adenosine, which have not been reported in previous studies.

Metabolic disorders of purines and pyrimidines are autosomal recessive inheritance disorders (41). There are three defects associated with pyrimidines, namely dihydropyrimidine dehydrogenase, dihydropyrimidinase and 3 -ureidopropionase deficiencies $(42,43)$. There are numerous purine-associated disorders, including orotic aciduria $(44,45)$. When considering biomarkers for these disorders, it is notable that thymine, 5-hydroxymethyl-uracil, dihydrouracil, dihydrothymine and uracil are characterized by poor solubility in organic solvents and so they have not been accurately tested using conventional methods. Furthermore, orotate is oxidized and reduced readily in pretreated urine to form, for example, $\beta$-ureidopropionate, $\beta$-ureidoisobutyrate, $\beta$-aminoisobutyrate, and $\beta$-alanine. Some of these changes could be classified as secondary purine and pyrimidine metabolic disorders, as the previously described OTC deficiency increases orotate levels.

Using the two-step derivatization method of the present study, concentrations of thymine, 5-hydroxymethyl-uracil, $\beta$-ureidopropionate, $\beta$-ureidoisobutyrate and orotate in patients could be detected at concentration ranges up to a hundred-fold those of normal controls, with a PCA model constructed using thymine, $\beta$-ureidopropionate and uracil, respectively.

Thus, the present method was found to be helpful for the analysis of purine and pyrimidine deficiencies. So far, we have examined only 3 cases with thymine-uracil aciduria combined with $\beta$-ureidopropionase deficiency (c.C216C/T and c.G977G/A), two cases with thymine-uracil aciduria (c.C216C/T), two cases with $\beta$-ureidopropionase deficiency (c.C792C/A combined c.G977G/A), and two cases with orotic aciduria to be examined. However, the prevalence of these metabolic disorders in Europe is reportedly only 26/3.2 million (46-48), suggesting that conventional methods by urine could have been affected in the course of investigation and thus lead to a false negative or incorrect result, which requires further investigation. Therefore, urine samples were pretreated at room temperature, and fine $\mathrm{pH}$-value variation was required to obtain the optimal conditions.

Mitochondrial metabolic disorders belong to the maternal or Mendelian inherited class of diseases $(49,50)$. Approximately $50 \%$ of patients succumb to these disorders by the age of 3 years. Mutations associated with Leigh syndrome 
are located in genes including MT-ATP6, MT-ND2 and MT-CO3 by Mendelian inheritance $(51,52)$. It has been reported that $10-20 \%$ patients with Leigh syndrome have a MT-ATP6 m.8993T>G mutation (53). When there is a severe defect of the complex IV respiratory chain enzyme of the mitochondrial oxidative phosphorylation system, the enzyme cannot convert ADP into ATP successfully as that of a healthy individual would. Thus, a chronic progressive disorder of mitochondrial energy metabolism occurs, with a series of clinical manifestations of mitochondrial energy $(54,55)$. Notably, estimable biomarkers for mitochondrial metabolic disorders remain to be verified; metabolites such as lactate, pyruvate and the lactate:pyruvate ratio; $\beta$-hydroxybutyrate, acetoacetate and the $\beta$-hydroxybutyrate:acetoacetate ratio, fumarate, malate, citrate, aconitate, ethylmalonate, methylmalonate, adipate, azelate, sebacate and 4-hydroxyphenyllactate have been reported in urine (21). In particular, many of the aforementioned changes might be attributable to secondary mitochondrial metabolic disorders (for example, vitamin B2 or vitamin B1 deficiency, valproate hyperlipidemia or propionic acidemia).

Despite the lack of specificity in qualitative parameters, findings for quantitative parameters identified markedly increased concentrations of $\alpha-\mathrm{KG}$ and succinate using the present two-step derivatization method. These two biomarkers have also been reported in previous studies $(56,57)$; however, they cannot be verified as biomarkers for mitochondrial metabolic disorders in the present study, due to the limited number of cases $(n=31)$. Therefore, they require further investigation. In the 31 cases with mitochondrial metabolic disorders (including m..8993T $>\mathrm{G}$, m.10191T> C, m.14487 T>C, m.14693 A > G and CoQ10 deficiency), the two-step derivatization process of the present study revealed that concentrations of $\alpha-K G$ alone or combined with succinate in untreated patients could be $>5$ to 130 -fold higher than those in normal controls, based on the construction of a PCA model using $\alpha-\mathrm{KG}(<5$-fold) and combined succinate with $\alpha-K G(>5$ to 130 -fold). It should be noted that their levels were not notably decreased following the administration of conventional vitamins.

In the quantitation procedure, the oximation processing time of $\alpha-K G$ was clearly reduced. Notably, $\alpha-K G$ has not previously been precisely examined using conventional MS-based methods with oximation in urine as it is thermally unstable, and readily undergoes oxidation and reduction. The present study resolved this technical problem using our own oximation reagents; thus, $\alpha-K G$ required only $<3 \mathrm{~min}$ for oximation in the urine samples, whereas, oximation in previous studies has been reported to take from 4 to $48 \mathrm{~h}$ (19). Also, it is widely reported that $\alpha$-KG is converted at least partially to $\alpha$-hydroxyglutarate during the conventional pretreatment used for urinary organic acids (58), as its oxidative and reductive reactions are increased under heating at higher $\mathrm{pH}$-values. Under the optimal conditions in the present study, oximation was conducted at room temperature for $<3 \mathrm{~min}$, as described in detail in our previous two studies $(8,20)$.

Biochemical substances that contain $\alpha$-KG, glyoxylate and other $\alpha$-keto acids and aldehydes have extremely unstable structures (59). Ion exchange, organic extraction, urease pretreatment and modified urease pretreatment methods in the analysis of urine have not been adapted to traditional oximation, and it is difficult to obtain stable GC/MS data for these categories of substances in the laboratory. However, the traditional method of oximation requires high temperatures and a relatively long reaction time, which greatly affects the combined analysis of semi-volatile, readily decomposable substances. Therefore, only $\alpha-\mathrm{KG}$ was used as an example to evaluate the method.

In conclusion, urine analysis with two-step derivatization has demonstrated an improved ability for the analysis of thermally unstable amino acids, $\alpha$-ketoacids, $\alpha$-aldehyde acids, and volatile and semi-volatile organic acids, which expands the range of compounds able to be simultaneously analyzed by GC/MS. In China, the novel technology described in this study has been applied effectively for the clinical screening and monitoring of five types of small molecule in children with IMDs. It has also provided a more comprehensive and reliable biochemical analysis tool than any previously used in China. Furthermore, it has provided an effective and reliable monitoring platform for use metabolomic studies of mitochondrial and nutritional metabolic disorders.

\section{Acknowledgements}

This work was supported by Hunan Province Technical Institute of Clinical Preventive and Treatment for Children's Inherited Metabolic Disorders. This work was financially supported by Hunan Province Department of Science and Technology (grant nos. 2013XC83-11, 2014SK4044 and 2015SK2032).

\section{References}

1. Pampols T: Inherited metabolic rare disease. Adv Exp Med Biol 686: 397-431, 2010.

2. Pérez B, Rodriguez-Pascau L, Vilageliu L, Grinberg D, Ugarte M and Desviat LR: Present and future of antisense therapy for splicing modulation in inherited metabolic disease. J Inherit Metab Dis 33: 397-403, 2010.

3. Rodrigues JV, Henriques BJ, Lucas TG and Gomes CM: Cofactors and metabolites as protein folding helpers in metabolic diseases. Curr Top Med Chem 12: 2546-2559, 2012.

4. Cassiman D: Gene transfer for inborn errors of metabolism of the liver: The clinical perspective. Curr Pharm Des 17: 2550-2557, 2011.

5. Blau N, Duran M, Gibson KM and Dionisi-Vici C (eds): Physician's Guide to the Diagnosis, Treatment, and Follow-Up of Inherited Metabolic Diseases. Springer, Heidelberg, 2014.

6. Song SM, Yoon HR, Lee A and Lee KR: Seven-year experience with inherited metabolic disorders screening by tandem mass spectrometry. J Genet Med 5: 21-25, 2008.

7. Janeckova H, Kalivodova A, Najdekr L, Friedecky D, Hron K, Bruheim P and Adam T: Untargeted metabolomic analysis of urine samples in the diagnosis of some inherited metabolic disorders. Biomed Pap Med Fac Univ Palacky Olomouc Czech Repub 159: 582-585, 2015.

8. Xiong X, Sheng X, Liu D, Zeng T, Peng Y and Wang Y: A GC/MS-based metabolomic approach for reliable diagnosis of phenylketonuria. Anal Bioanal Chem 407: 8825-8833, 2015.

9. Kuhara T: Diagnosis of inborn errors of metabolism using filter paper urine, urease treatment, isotope dilution and gas chromatography-mass spectrometry. J Chromatogr B Biomed Sci Appl 758: 3-25, 2001.

10. Kuhara T: Diagnosis and monitoring of inborn errors of metabolism using urease-pretreatment of urine, isotope dilution, and gas chromatography-mass spectrometry. J Chromatogr B Analyt Technol Biomed Life Sci 781: 497-517, 2002.

11. Kuhara T, Ohse M, Inoue Y, Yorifuji T, Sakura N, Mitsubuchi H, Endo F and Ishimatu J: Gas chromatographic-mass spectrometric newborn screening for propionic acidaemia by targeting methylcitrate in dried filter-paper urine samples. J Inherit Metab Dis 25: 98-106, 2002.

12. Fryčák P, Lemr K, Adam T and Hušková R: Diagnostics of some inherited metabolic disorders by mass spectrometry using modern ionisation techniques. Chemicke Listy 97: 93-100, 2003. 
13. Bruheim P, Kvitvang HF and Villas-Boas SG: Stable isotope coded derivatizing reagents as internal standards in metabolite profiling. J Chromatogr A 1296: 196-203, 2013.

14. Bouatra S, Aziat F, Mandal R, Guo AC, Wilson MR, Knox C, Bjorndahl TC, Krishnamurthy R, Saleem F, Liu P, et al: The human urine metabolome. PLoS One 8: e73076, 2013.

15. Janečková $H$, Hron K, Wojtowicz P, Hlídková E, Barešová $\mathrm{A}$, Friedecký D, Zídková L, Hornik P, Behúlová D, Procházková $\mathrm{D}$, et al: Targeted metabolomic analysis of plasma samples for the diagnosis of inherited metabolic disorders. J Chromatogr A 1226: 11-17, 2012.

16. Qun G: Evaluation of automated sample preparation, retention time locked GC-MS and automated data analysis for the metabolomic study of Arabidopsis species. J Chromatogr A 1218: 3247-3254, 2010.

17. Scher HI, Nasso SF, Rubin EH and Simon R: Adaptive clinical trial designs for simultaneous testing of matched diagnostics and therapeutics. Clin Cancer Res 17: 6634-6640, 2011.

18. Rosser CJ, Dai Y, Miyake M, Zhang G and Goodison S: Simultaneous multi-analyte urinary protein assay for bladder cancer detection. BMC Biotechnol 14: 81-89, 2013.

19. Wang YC: A two-step derivatization on simultaneous analysis for organic acids, fatty acids, amino acid, carbohydrates, pyrimidines, and purines in complicated organic compounds. CN patent ZL201210114246.2. Filed April 18, 2012; issued December 18, 2013.

20. Xiong X, Liu D, Wang Y, Zeng $\mathrm{T}$ and Peng $\mathrm{Y}$ : Urinary 3-(3-hydroxyphenyl)-3-hydroxypropionic acid, 3-hydroxyphenylacetic acid, and 3-hydroxyhippuric acid are elevated in children with autism spectrum disorders. Biomed Research International 2016: 9485412, 2016.

21. Blau N, Duran M, Blaskovics ME and Gibson MK (eds): Physician's Guide to the Laboratory Diagnosis of Metabolic Diseases. 2nd edition. Chapman and Hall Medical, London, 1996.

22. Xiong $\mathrm{X}$, Liu D, Wang $\mathrm{Y}$, Zeng $\mathrm{T}$ and Peng Y: Urinary 3-(3-hydroxyphenyl)-3-hydroxypropionic acid, 3-hydroxyphenylacetic acid, and 3-hydroxyhippuric acid are elevated in children with autism spectrum disorders. BioMed Res Int 2016: 1-8, 2016.

23. Trujillano D, Perez B, González J, Tornador C, Navarrete R, Escaramis E, Ossowski S, Armengol L, Cornejo V, Desviat LR, et al: Accurate molecular diagnosis of phenylketonuria and tetrahydrobiopterin-deficient hyperphenylalaninemias using high-throughput targeted sequencing. Eur J Hum Genet 22: 528-534, 2014.

24. Setoodeh A, Yarali B, Rabbani A, Khatami S and Shams S: Tetrahydrobiopterin responsiveness in a series of 53 cases of phenylketonuria and hyperphenylalaninemia in Iran. Mol Genet Metab Rep, 2:77-79, 2015

25. Okano Y, Kudo S, Nishi Y, Sakaguchi T and Aso K: Molecular characterization of phenylketonuria and tetrahydrobiopterin-responsive phenylalanine hydroxylase deficiency in Japan. J Hum Genet 56: 306-312, 2011.

26. Yaplito-Lee J, Chow CW and Boneh A: Histopathological findings in livers of patients with urea cycle disorders. Mol Genet Metab 108: 161-165, 2013

27. Nagasaka H, Yorifuji T, Egawa H, Inui A, Fujisawa T, Komatsu H, Tsukahara $\mathrm{H}$, Uemoto $\mathrm{S}$ and Inomata Y: Characteristics of NO cycle coupling with urea cycle in non-hyperammonemic carriers of ornithine transcarbamylase deficiency. Mol Genet Metab 109: 251-254, 2013

28. Mukhtar A, Dabbous H, El Sayed R, Aboulfetouh F, Bahaa M, Abdelaal A, Fathy M and El-Meteini M: A novel mutation of the ornithine transcarbamylase gene leading to fatal hyperammonemia in a liver transplant recipient. Am J Transplant 13: 1084-1087, 2013.

29. Paine SM, Grünewald S and Jacques TS: Antenatal neurodevelopmental defects in ornithine transcarbamylase deficiency. Neuropathol Appl Neurobiol 38: 509-512, 2012.

30. Mhanni AA, Prasad C and Rockman-Greenberg C: Ornithine transcarbamylase deficiency presenting as recurrent abdominal pain in childhood. Pediatr Emerg Care 27: 850-853, 2011

31. Swarts L, Leisegang F, Owen EP and Henderson HE: An OTC deficiency 'phenocopy' in association with Klinefelter syndrome. J Inherit Metab Dis 30: 101, 2007

32. Wang LL, Morizono H, Lin JP, Bell P, Jones D, McMenamin D, Yu HW, Batshaw ML and Wilson JM: Preclinical evaluation of a clinical candidate AAV8 vector for Ornithine Transcarbamylase (OTC) deficiency reveals functional enzyme from each persisting vector genome. Mol Genet Metab 105: 203-211, 2012.
33. Vaidyanathan K: Molecular diagnosis of urea cycle disorders: Current global scenario. Indian J Biochem Biophys 50: 357-362, 2013.

34. Kobayashi K, Sinasac DS, Iijima M, Boright AP, Begum L, Lee JR, Yasuda T, Ikeda S, Hirano R, Terazono H, et al: The gene mutated in adult-onset type II citrullinaemia encodes a putative mitochondrial carrier protein. Nat Genet 22: 159-163, 1999.

35. Contreras L, Gomez-Puertas P, Iijima M, Kobayashi K, Saheki T and Satrustegui J: $\mathrm{Ca}^{2+}$ Activation kinetics of the two aspartate-glutamate mitochondrial carriers, aralar and citrin: Role in the heart malate-aspartate NADH shuttle. J Biol Chem 282: 7098-7106, 2007.

36. Saheki T, Iijima M, Li MX, Kobayashi K, Horiuchi M, Ushikai M, Okumura F, Meng XJ, Inoue I, Tajima A, et al: Citrin/ mitochondrial glycerol-3-phosphate dehydrogenase double knock-out mice recapitulate features of human citrin deficiency. J Biol Chem 282: 25041-25052, 2007.

37. Song YZ, Li BX, Chen FP, Liu SR, Sheng JS, Ushikai M, Zhang $\mathrm{CH}$, Zhang T, Wang ZN, Kobayashi K, et al: Neonatal intrahepatic cholestasis caused by citrin deficiency: Clinical and laboratory investigation of 13 subjects in mainland of China. Dig Liver Dis 41: 683-689, 2009.

38. Yamaguchi N, Kobayashi K, Yasuda T, Nishi I, Iijima M, Nakagawa M, Osame M, Kondo I and Saheki T: Screening of SLC25A13 mutations in early and late onset patients with citrin deficiency and in the Japanese population: Identification of two novel mutations and establishment of multiple DNA diagnosis methods for nine mutations. Hum Mutat 19: 122-130, 2002

39. Zhang MH, Gong JY and Wang JS: Citrin deficiency presenting as acute liver failure in an eight-month-old infant. World $\mathrm{J}$ Gastroenterol 21: 7331-7334, 2015.

40. Wang LY, Chen NI, Chen PW, Chiang SC, Hwu WL, Lee NC and Chien YH: Newborn screening for citrin deficiency and carnitine uptake defect using second-tier molecular tests. BMC Med Genet 14: 1-6, 2013.

41. Wert SE, Whitsett JA and Nogee LM: Genetic disorders of surfactant dysfunction. Pediatr Dev Pathol 12: 253-274, 2009.

42. Chen BC, Mohd Rawi R, Meinsma R, Meijer J, Hennekam RC and van Kuilenburg AB: Dihydropyrimidine dehydrogenase deficiency in two malaysian siblings with abnormal MRI findings. Mol Syndromol 5: 299-303, 2014.

43. Chang HS, Shibata T, Arai S, Zhang CH, Yabuki A, Mitani S, Higo T, Sunagawa K, Mizukami $\mathrm{K}$ and Yamato $\mathrm{O}$ : Dihydropyrimidinase deficiency: The first feline case of dihydropyrimidinuria with clinical and molecular findings. JIMD Rep 6: 21-26, 2012.

44. Micheli V, Camici M, Tozzi MG, Ipata PL, Sestini S, Bertelli M and Pompucci G: Neurological disorders of purine and pyrimidine metabolism. Curr Top Med Chem 11: 923-947, 2011.

45. Porcu S, Corda M, Lilliu F, Contini L, Era B, Traldi P and Fais A: Increase in urinary purines and pyrimidines in patients with methylmalonic aciduria combined with homocystinuria. Clin Chim Acta 411: 853-858, 2010.

46. Nakajima Y, Meijer J, Dobritzsch D, Ito T, Meinsma R, Abeling NGG, Roelofsen J, Zoetekouw L, Watanabe Y,Tashiro K, et al: Clinical, biochemical and molecular analysis of 13 Japanese patients with $\beta$-ureidopropionase deficiency demonstrates high prevalence of the c. $977 \mathrm{G}>\mathrm{A}$ (p.R326Q) mutation. J Inherit Metab Dis 37: 801-812, 2014.

47. Jurecka A. Inborn errors of purine and pyrimidine metabolism. J Inherit Metab Dis 32: 247-263, 2009.

48. Balasubramaniam S, Duley JA and Christodoulou J: Inborn errors of pyrimidine metabolism: clinical update and therapy. J Inherit Metab Dis 37: 687-698, 2014.

49. Ruhoy IS and Saneto RP: The genetics of Leigh syndrome and its implications for clinical practice and risk management. Appl Clin Genet 7: 221-234, 2014

50. Honzik T, Tesarova M, Vinsova K, Hansikova H, Magner M, Kratochvilova H, Zamecnik J, Zeman J and Jesina P: Different laboratory and muscle biopsy findings in a family with an m.8851T>C mutation in the mitochondrial MTATP6 gene. Mol Genet Metab 108: 102-105, 2013.

51. Hejzlarová K, Kaplanová V, Nůsková H, Kovářová N, Ješina P, Drahota Z, Mráček T, Seneca S and Houštěk J: Alteration of structure and function of ATP synthase and cytochrome c oxidase by lack of Fo-a and Cox3 subunits caused by mitochondrial DNA 9205delTA mutation. Biochem J 466: 601-611, 2015 . 
52. Chol M, Lebon S, Bénit P, Chretien D, de Lonlay P, Goldenberg A, Odent S, Hertz-Pannier L, Vincent-Delorme C, Cormier-Daire $\mathrm{V}$, et al: The mitochondrial DNA G13513A MELAS mutation in the NADH dehydrogenase 5 gene is a frequent cause of Leigh-like syndrome with isolated complex I deficiency. J Med Genet 40: 188-191, 2003.

53. Kara B, Arikan M, Maraş H, Abacı N, Cakıris A and Ustek D: Whole mitochondrial genome analysis of a family with NARP/ MILS caused by m.8993T>C mutation in the MT-ATP6 gene. Mol Genet Metab 107: 389-393, 2012.

54. Anglin RE, Garside SL, Tarnopolsky MA, Mazurek MF and Rosebush PI: The psychiatric manifestations of mitochondrial disorders: A case and review of the literature. J Clin Psychiatry 73 506-512, 2012.

55. Hulgan T, Haubrich R, Riddler SA, Tebas P, Ritchie MD, McComsey GA, Haas DW and Canter JA: European mitochondrial DNA haplogroups and metabolic changes during antiretroviral therapy in AIDS Clinical Trials Group Study A5142. AIDS 25: 37-47, 2011.
56. Xiao MT, Yang H, Xu W, Ma SH, Lin HP, Zhu HG, Liu LX, Liu Y, Yang C, Xu YH, et al: Inhibition of $\alpha$-KG-dependent histone and DNA demethylases by fumarate and succinate that are accumulated in mutations of FH and SDH tumor suppressors. Genes Dev 29: 1326-1338, 2012.

57. MacKenzie ED, Selak MA, Tennant DA, Payne LJ, Crosby S, Frederiksen CM, Watson DG and Gottlieb E: Cell-permeating $\alpha$-ketoglutarate derivatives alleviate pseudohypoxia in succinate dehydrogenase-deficient cells. Mol Cell Biol 27: 3282-3289, 2007.

58. Leonardi R, Subramanian C, Jackowski S and Rock CO: Cancer-associated isocitrate dehydrogenase mutations inactivate NADPH-dependent reductive carboxylation. J Biol Chem 287: 14615-14620, 2012.

59. Baughn AD, Garforth SJ, Vilchèze C and Jacobs WR Jr: An anaerobic-type alpha-ketoglutarate ferredoxin oxidoreductase completes the oxidative tricarboxylic acid cycle of Mycobacterium tuberculosis. PLoS Pathog 5: e1000662, 2009. 